\title{
Stable ultra-thin CdTe crystal: a robust direct gap semiconductor
}

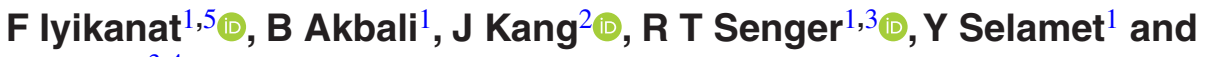 \\ H Sahin ${ }^{3,4}$ (D) \\ 1 Department of Physics, Izmir Institute of Technology, 35430, Izmir, Turkey \\ 2 Materials Sciences Division, Lawrence Berkeley National Laboratory, Berkeley, CA 94720, \\ United States of America \\ 3 ICTP-ECAR Eurasian Center for Advanced Research, Izmir Institute of Technology, 35430, Izmir, \\ Turkey \\ ${ }^{4}$ Department of Photonics, Izmir Institute of Technology, 35430, Izmir, Turkey
}

E-mail: fadiliyikanat@iyte.edu.tr and hasansahin@iyte.edu.tr

Received 8 August 2017, revised 14 October 2017

Accepted for publication 24 October 2017

Published 9 November 2017

\begin{abstract}
Employing density functional theory based calculations, we investigate structural, vibrational and strain-dependent electronic properties of an ultra-thin CdTe crystal structure that can be derived from its bulk counterpart. It is found that this ultra-thin crystal has an 8-atom primitive unit cell with considerable surface reconstructions. Dynamic stability of the structure is predicted based on its calculated vibrational spectrum. Electronic band structure calculations reveal that both electrons and holes in single layer CdTe possess anisotropic in-plane masses and mobilities. Moreover, we show that the ultra-thin CdTe has some interesting electromechanical features, such as strain-dependent anisotropic variation of the band gap value, and its rapid increase under perpendicular compression. The direct band gap semiconducting nature of the ultra-thin CdTe crystal remains unchanged under all types of applied strain. With a robust and moderate direct band gap, single-layer CdTe is a promising material for nanoscale strain dependent device applications.
\end{abstract}

Keywords: first principle calculations, II-VI materials, direct gap semiconductors, ultra-thin materials

(Some figures may appear in colour only in the online journal)

\section{Introduction}

Discovery of graphene [1] has attracted great interest towards the family of two-dimensional (2D) crystal structures. In addition to graphene, other 2D crystals such as germanene [2, 3], silicene $[4,5]$, stanene $[6,7]$, transition-metal dichalcogenides (TMDs), [8-12] and post-transition-metal chalcogenides (TMCs) [13-15] have been predicted and successfully synthesized. One of the most prominent members of $2 \mathrm{D}$ crystals is $\mathrm{MoS}_{2} . \mathrm{MoS}_{2}$ exhibits a transition from an indirect band gap of $1.29 \mathrm{eV}$ to a direct band gap of $1.90 \mathrm{eV}$ when its layer thickness is reduced from bulk to a single-layer $[16,17]$. Singlelayer $\mathrm{MoS}_{2}$ based field-effect transistors (FETs) can have

${ }^{5}$ Author to whom any correspondence should be addressed. room-temperature on/off ratios of the order of $10^{8}$ and these fabricated transistors can exhibit a carrier mobility larger than $200 \mathrm{~cm}^{2}(\mathrm{~V} \mathrm{~s})^{-1}[18,19]$. Moreover, $\mathrm{MoS}_{2}$ has excellent mechanical properties like high flexibility $[20,21]$ and high strength [22]. Due to these outstanding properties, 2D materials will play an important role in the applications of future optoelectronics and flexible electronics.

Recent studies have shown that not only layered materials but also ultra-thin forms of non-layered materials that consist of a few atomic layer thickness can form 2D crystals $[4,23,24]$. For instance, CdSe, CdS and CdTe nanoplatelets with thicknesses ranging from 4 to 11 monolayers were synthesized [25]. The thickness dependence of the absorption and emission spectra of these nanoplatelets were demonstrated. Park et al achieved successful synthesis of $1.4 \mathrm{~nm}$-thick ZnSe 
nanosheets with wurtzite structure [26]. Using a colloidal template method large-scale fabrication of free-standing ultrathin and lamellar-structured CdSe with wurtzite crystal structure was achieved [27]. Furthermore, using a lamellar hybrid intermediate, large-area, free-standing, single-layers of $\mathrm{ZnSe}$ were fabricated [28]. Single-layers of $\mathrm{ZnSe}-$ pa (pa stands for n-propylamine) were exfoliated from a lamellar hybrid $\left(\mathrm{Zn}_{2}\right.$ $\mathrm{Se}_{2}$ ) (pa) intermediate. Then, by heat treatment pa-molecules were cleared off and the colloidal suspension of clean $\mathrm{ZnSe}$ single-layers was obtained. Fabricated single-layer $\mathrm{ZnSe}$ has four-atomic-layer thickness. They showed that, produced single-layer ZnSe was highly stable over several days. The photocurrent densities of these monolayers are much higher than that of their bulk counterparts.

Cadmium telluride (CdTe) is one of the most popular II-VI semiconductors because of its potential applications in optoelectronic devices such as photodetectors, solar cells and room temperature X-and gamma-ray detectors [29-32]. CdTe has a direct optical band gap of $\sim 1.5 \mathrm{eV}$ with a high absorption coefficient $[33,34]$. Solar cell efficiency of CdTe-based thin-films has recently reached $22.1 \%$ [35]. CdTe crystallizes in the zinc-blende structure at room temperature. The CdTe thin films can be grown by various deposition techniques such as chemical vapor deposition [36], pulsed laser deposition [37], electrochemical deposition [38] and spray pyrolysis [39]. Generally, the intrinsic properties of ultra-thin materials exhibit drastic changes compared to their bulk counterparts. Thus, when a material is thinned from bulk to ultra-thin form, it can exhibit enhanced properties and new functionalities.

In this study, motivated by the recent synthesis of ultrathin II-VI binary compounds, we investigate structural, electronic and vibrational properties of single-layer CdTe using first principle calculations based on density functional theory (DFT). Although there are a few prior computational studies on single-layer CdTe [40, 41], free-standing monolayer CdTe has not been predicted yet. We found that single-layer CdTe containing eight atoms in the primitive unit cell is structurally stable with anisotropic electronic properties. It has a direct band-gap at the $\Gamma$ point and direct gap transition at the $\Gamma$ point is not affected by strain along any direction. The straindependent anisotropic variation of the band gap value and its rapid increase under out-of-plane compression pressure are found. The paper is organized as follows: details of the computational methodology are given in section 2. Structural and electronic properties of single-layer CdTe are presented in section 3. In section 4, the dynamical stability of single-layer CdTe is studied. Effect of strain on electronic properties is discussed in section 5. Finally, we outline our results in section 6.

\section{Computational methodology}

All calculations were performed within the density functional theory (DFT) using projector-augmented-wave potentials (PAW) and a plane-wave basis set as implemented in the Vienna ab initio simulation package (VASP) [42, 43]. Perdew-Burke-Ernzerhof (PBE) version of generalized gradient approximation (GGA) [44] was used for the description of the exchange-correlation functional. Analysis of the charge transfers in the structures was made by the Bader technique [45]. The ionization energy is determined as the energy difference between the valance band maximum energy and the vacuum level at the (110) side of the bulk and single-layer CdTe.

The conjugate gradient algorithm was used to optimize the structure. The cutoff energy for the plane-waves was chosen to be $500 \mathrm{eV}$. The convergence criterion for energy was taken to be $10_{-}-5 \mathrm{eV}$ between two consecutive steps. The convergence for the Hellmann-Feynman force in each unit cell was taken to be $10^{-4} \mathrm{eV} \AA^{-1}$. The pressure in the unit cell was kept below $1 \mathrm{kBar}$. In order to eliminate interlayer interaction within the periodic images, a vacuum spacing of approximately $12 \AA$ between adjacent layers was chosen. For the structural optimization, a $9 \times 12 \times 1 \Gamma$-centered $k$-point mesh was used. The cohesive energy per atom was calculated using the formula

$$
E_{c}=\left[n_{\mathrm{Cd}} E_{\mathrm{Cd}}+n_{\mathrm{Te}} E_{\mathrm{Te}}-E_{\mathrm{SL}}\right] / n
$$

where $E_{\mathrm{Cd}}$ and $E_{\mathrm{Te}}$ are isolated single atom energies for $\mathrm{Cd}$ and $\mathrm{Te}$, respectively. While $n$ stands for the number of all atoms, $n_{\mathrm{Cd}}$ and $n_{\mathrm{Te}}$ show the numbers of $\mathrm{Cd}$ and Te atoms in the unit cell, respectively. $E_{\mathrm{SL}}$ denotes the total energy of the single-layer CdTe. Phonon dispersions and eigenvectors are calculated by making use of the small displacement methodology implemented in the PHON code [46].

\section{Structural and electronic properties of single-layer CdTe}

It is well-known that the bonding character of zinc-blende CdTe is partly covalent and partly ionic [47, 48]. Except for the (1 1 0$)$ facets, zinc-blende structure of CdTe has polar surfaces, which are chemically highly active. Even if single-layer structures having these polar surfaces could be obtained, their chemical activity would hinder their stability. However, since the (110) surfaces are non-polar, cleavage along these planes could be more feasible.

The proposed structure of CdTe single-layers in our study have the same crystal structure as the fabricated highly stable single-layer of zinc-blende $\mathrm{ZnSe}(z b-\mathrm{ZnSe})$ [28]. Side views along $\vec{a}$ and $\vec{b}$ directions and top view of single-layer CdTe are shown in figures 1(a)-(c), respectively. Lattice parameters of single-layer CdTe are found to be $a=6.18$ and $b=4.53 \AA$. Calculated lattice parameters are smaller than those for bulk CdTe which is $6.52 \AA$. Figure 1 (a) shows that the $\mathrm{Cd}-\mathrm{Te}$ bond lengths vary from 2.77 to $2.90 \AA$, bond lengths between surface atoms being smaller than those of the inner atoms in the layer. As seen in figure 1(b), Te atoms are at the surfaces of the layer, and each surface Te atom binds to three $\mathrm{Cd}$ atoms. The inner Te atoms are surrounded by $4 \mathrm{Cd}$ atoms with tetrahedral type bonds. During the atomic relaxation of the truncated layer, $\mathrm{Cd}$ atoms that are at the surface recede toward the inner Te atoms; remaining Te atoms move outward. Such reconstructions stabilize the layer surfaces.

Bader charge analysis reveals that each $\mathrm{Cd}$ atom donates $0.5 e$ to each Te atom. To illustrate the charge transfer mechanism three dimensional charge density differences are shown in figure 1(d). The charge density differences were calculated 

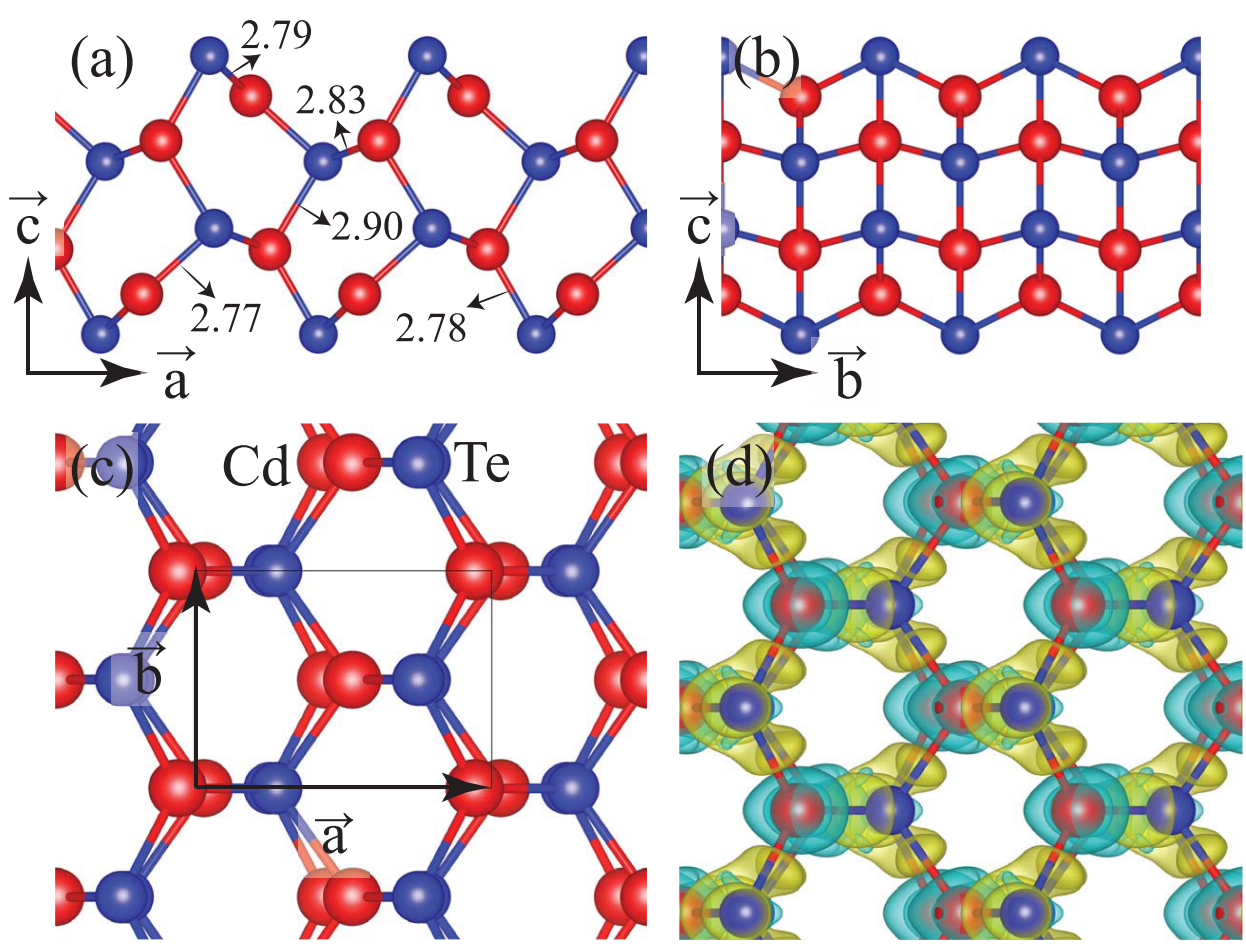

Figure 1. Side views (a) along $\vec{a}$ lattice vector, (b) along $\vec{b}$ lattice vector and (c) top view of single-layer CdTe. Black lines represent the rectangular unit cell. (d) The charge densities of the isolated $\mathrm{Cd}$ and Te atoms are subtracted from the charge density of single-layer CdTe. The yellow and blue densities stand for the negative and positive charges, respectively. Red and blue atoms are for Cd and Te, respectively.

by subtracting charge of isolated $\mathrm{Cd}$ and $\mathrm{Te}$ atoms from charge of single-layer CdTe. The charge transfer between $\mathrm{Cd}$ and Te atoms resembles polar-covalent bonding. Due to a difference in the electronegativities of $\mathrm{Cd}$ and Te atoms (1.69 and 2.10 for $\mathrm{Cd}$ and $\mathrm{Te}$ atoms, respectively), the $\mathrm{Cd}-\mathrm{Te}$ bonding has also some ionic character. Finally, the cohesive energy per atom of single-layer CdTe is $1.79 \mathrm{eV}$ which is less than the bulk value of $2.20 \mathrm{eV}$ per atom.

To investigate the full band dispersions and the characteristics of band edges in the Brillouin zone (BZ), whole BZ energy-band structure is calculated and given in figure 2(a). As shown in the figure, valence band maximum (VBM) and conduction band minimum (CBM) of CdTe reside at the same symmetry point of the $\Gamma$. The calculated GGA electronic-band structure demonstrate that single-layer CdTe is a direct-gap semiconductor with a band gap of $1.42 \mathrm{eV}$. In order to give more accurate gap energy of the single-layer CdTe the calculated band structures within HSE06 correction are also shown figure 2(a). A calculated HSE06 gap of single-layer CdTe is $2.13 \mathrm{eV}$. Since the trend and qualitative behavior of all the bands calculated using GGA and HSE06 are similar, only the GGA based results are given in the rest of the paper.

In order to properly understand the electronic properties of CdTe, partial density of states (PDOS) is also plotted in figure 2(b). The states in the vicinity of VBM are mostly composed of $p_{y}$ orbitals of Te. These $p_{y}$ orbitals of Te atoms are parallel to the $b$ lattice vector of the unit cell. On the other hand, CBM is mostly made up of the $s$ orbitals of $\mathrm{Cd}$ and the $s$ and $p_{z}$ orbitals of Te. Note that, the $p_{z}$ orbital contribution of Te atom mainly comes from surface Te atoms. Twodimensional contour plots of the valence band (VB) and the conduction band $(\mathrm{CB})$ of the single-layer CdTe are shown in figures 2(c) and (d). The directional anisotropy at the band edges is clearly seen in the surface plots. Ionization energy (I. E.) of single-layer and bulk CdTe surfaces are also calculated and are shown in table 1.

Due to reduced crystal symmetry in a single layer form of a material, its electronic characteristics are quite different from their bulk forms. Moreover, in-plane anisotropy in the ultrathin materials can lead to significant modifications in the electronic properties of the material. Therefore, the investigation of direction-dependent electronic properties of ultra-thin materials is of importance. The effective masses of electron $\left(m_{\mathrm{e}}\right)$ and hole $\left(m_{\mathrm{h}}\right)$ of single-layer CdTe are calculated near the $\Gamma$ point. Our calculations show that the $m_{\mathrm{e}}$ and $m_{\mathrm{h}}$ effective masses are highly anisotropic around the $\Gamma$ point. As given in table $2 m_{\mathrm{e}}$ values are $0.39,0.17$ and 0.21 for $\Gamma \rightarrow \mathrm{X}$, $\Gamma \rightarrow \mathrm{Y}$ and $\Gamma \rightarrow \mathrm{A}$, respectively. $m_{\mathrm{h}}$ values are $0.74,0.14$ and 0.20 for $\Gamma \rightarrow \mathrm{X}, \Gamma \rightarrow \mathrm{Y}$ and $\Gamma \rightarrow \mathrm{A}$, respectively. As seen in figure 2(b), the VBM is mainly composed of $p_{y}$ electrons of Te atoms, thus this causes a high in-plane anisotropy in $m_{\mathrm{h}}$ values. The anisotropy in the electron and hole masses are evident even from the crystal structure where $x$ - and $y$-directions are highly anisotropic (see figure 1). For comparison, the calculated values of $m_{\mathrm{e}}$ and $m_{\mathrm{h}}$ of bulk CdTe are also given in table 2 .

\section{Dynamical stability of single-layer CdTe}

Dynamical stability of the single-layer CdTe is investigated by examining the phonon spectra of the crystal. The small displacement method as implemented in the PHON software 

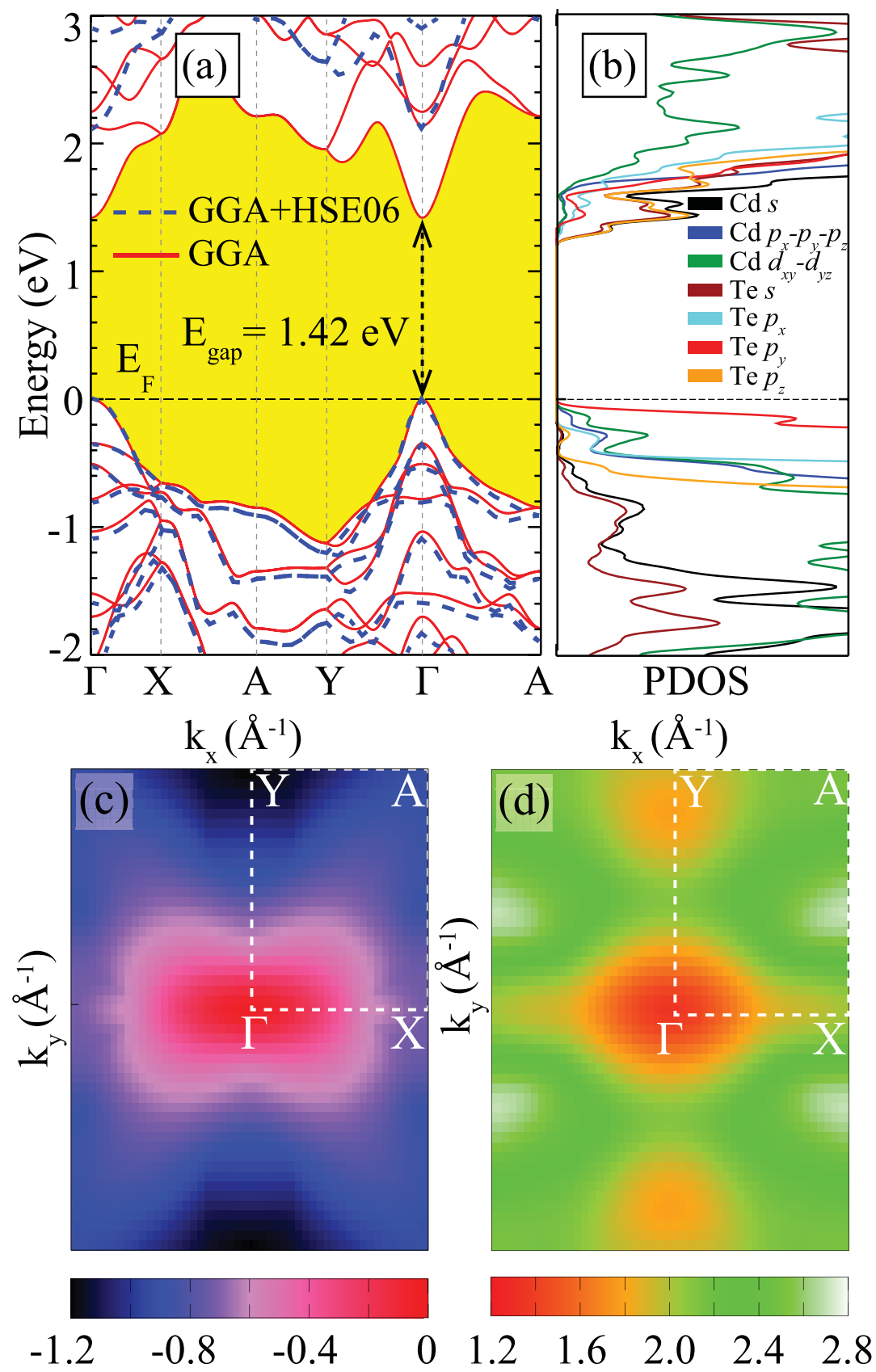

Figure 2. (a) Electronic band structure and (b) atom- and orbital-decomposed electronic density of states of single-layer CdTe. Fermi level is set to zero. (c) and (d) 2D surface plots of the valence band and the conduction band edges in the reciprocal space, respectively. The energy values $(\mathrm{eV})$ are color coded below the plots.

package is used to calculate the phonon spectra [46]. $4 \times 4 \times 1$ supercell is used for the phonon-band structure calculations. In figure 3 , we present the calculated phonon-band structure of single-layer CdTe obtained by the method described above. It is found that all the phonon modes have real eigenfrequencies, which indicate that CdTe single-layers are stable. The small imaginary frequencies (less than $1 \mathrm{~cm}^{-1}$ ) near the $\Gamma$ point are numerical artifacts caused by the inaccuracy of the FFT grid and they get cured as larger and larger supercells are considered.
Table 1. The calculated ground state properties for bulk and singlelayer (SL) CdTe: The lattice constants, $a$ and $b$; atomic distance between $\mathrm{Cd}$ and Te atoms, $d_{\mathrm{Cd}-\mathrm{Te}}$; charge transfer from $\mathrm{Cd}$ to Te atom, $\Delta \rho$; the cohesive energy per atom, $E_{c}$; energy band gap, $\mathrm{E}_{\text {gap }}$; and ionization energy, I. E.

\begin{tabular}{llllllll}
\hline & $\begin{array}{l}a \\
(\mathrm{~A})\end{array}$ & $\begin{array}{l}b \\
(\mathrm{~A})\end{array}$ & $\begin{array}{l}d_{\mathrm{Cd}-\mathrm{Te}} \\
(\AA)\end{array}$ & $\begin{array}{l}\Delta \rho \\
(e)\end{array}$ & $\begin{array}{l}E_{c} \\
(\mathrm{eV})\end{array}$ & $\begin{array}{l}E_{\text {gap }} \\
(\mathrm{eV})\end{array}$ & $\begin{array}{l}\mathrm{I} . \mathrm{E} . \\
(\mathrm{eV})\end{array}$ \\
\hline Bulk CdTe & 6.52 & - & 2.82 & 0.5 & 2.20 & 0.72 & 5.22 \\
SL CdTe & 6.18 & 4.53 & $2.77-2.90$ & 0.5 & 1.79 & 1.42 & 5.15 \\
\hline
\end{tabular}


Table 2. Effective masses of electrons $\left(m_{\mathrm{e}}\right)$ and holes $\left(m_{\mathrm{h}}\right)$ of bulk and single-layer (SL) CdTe.

\begin{tabular}{lllllllll}
\hline & $m_{\mathrm{e}}(\Gamma \rightarrow \mathrm{L})$ & $m_{\mathrm{e}}(\Gamma \rightarrow \mathrm{X})$ & $m_{\mathrm{e}}(\Gamma \rightarrow \mathrm{Y})$ & $m_{\mathrm{e}}(\Gamma \rightarrow \mathrm{A})$ & $m_{\mathrm{h}}(\Gamma \rightarrow \mathrm{L})$ & $m_{\mathrm{h}}(\Gamma \rightarrow \mathrm{X})$ & $m_{\mathrm{h}}(\Gamma \rightarrow \mathrm{Y})$ & $m_{\mathrm{h}}(\Gamma \rightarrow \mathrm{A})$ \\
\hline Bulk CdTe & 0.10 & 0.09 & - & - & 0.84 & 0.72 & - & - \\
SL CdTe & - & 0.39 & 0.17 & 0.21 & - & 0.74 & 0.14 & 0.20 \\
\hline
\end{tabular}

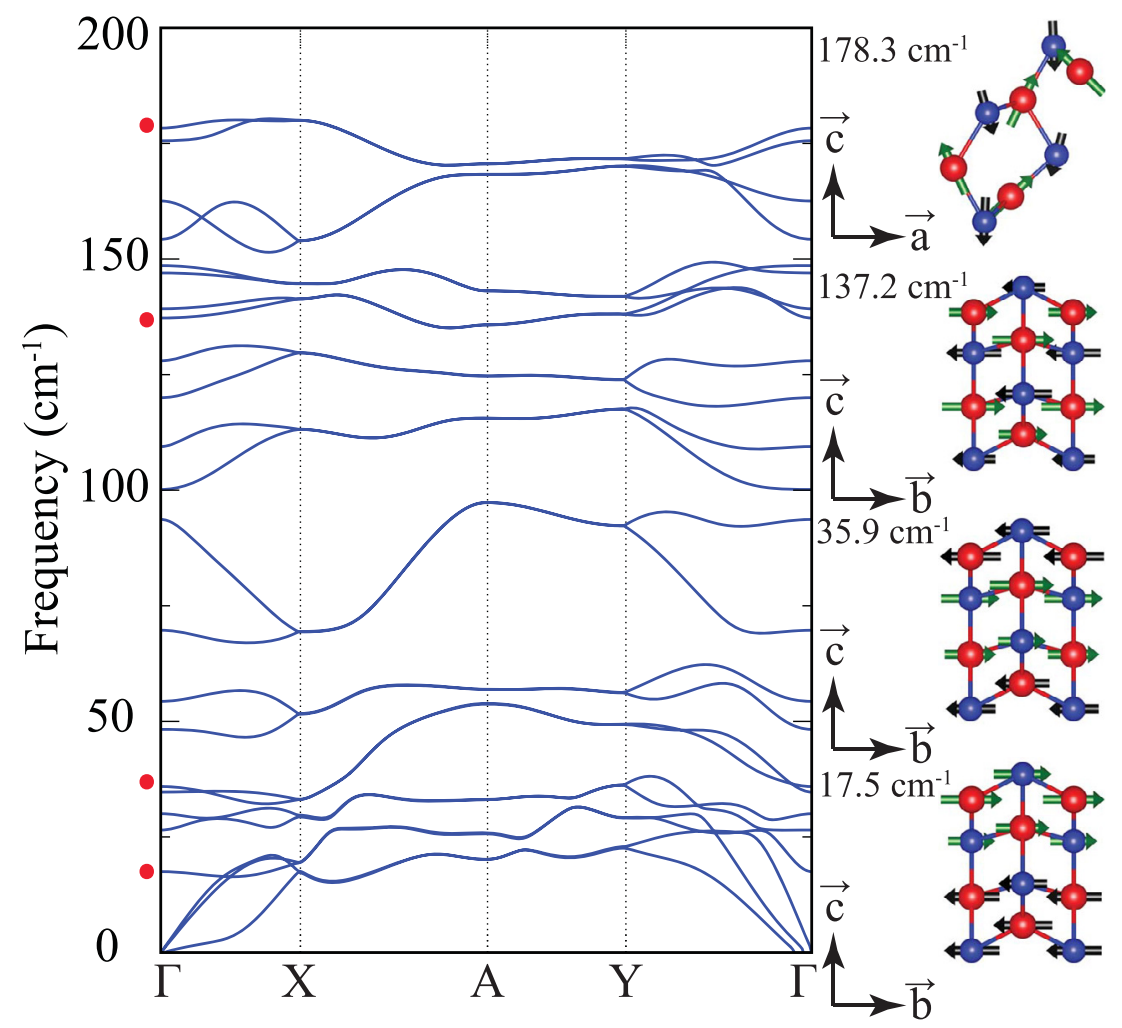

Figure 3. The phonon spectrum of monolayer CdTe is shown on the left. The branches of the possible Raman-active modes are indicated by the red dots and the corresponding normal modes are shown on the right.

The structural characteristics of bulk $z b-\mathrm{CdTe}$ were well studied in earlier Raman studies. The unit cell of bulk $z b-$ CdTe consists of one $\mathrm{Cd}$ and one $\mathrm{Te}$ atoms, therefore the phonon dispersion of bulk CdTe yields three acoustic and three optical modes. Main Raman active phonon modes are transverse optical (TO) and longitudinal optical (LO) modes and they occur approximately at 141 and $168 \mathrm{~cm}^{-1}$ [49]. In addition to these prominent modes, $\mathrm{A}_{1}$ and $\mathrm{E}$ symmetry modes were reported at $92,103,120$ and $147 \mathrm{~cm}^{-1}$ which give information about the presence of Te on the surface of bulk CdTe $[49,50]$.

On the other hand, the unit cell of single-layer CdTe contains four $\mathrm{Cd}$ atoms and four Te atoms. Therefore, the phonon dispersion of single-layer CdTe possesses three acoustic and twenty-one optical modes as shown in figure 3. As pointed out in the previous section there is a relaxation of the top atomic layers in the single-layer CdTe and bond length of the surface atoms is shorter than bond length of the inner atoms. Distortions of surface atoms lead to several flat phonon bands in figure 3. These distortions lift the degeneracies at the $\Gamma$ point and lead to hybridization of the acoustic and optical phonon branches. Optical character and frequency of possible Raman active modes are shown in the right panel of figure 3. The modes at $17.5,35.9$ and $137.2 \mathrm{~cm}^{-1}$ have in-plane character $\left(E_{g}\right.$ like) and the motion of the atoms are parallel to the $\vec{b}$. For the mode $137.2 \mathrm{~cm}^{-1}, \mathrm{Cd}$ and Te atoms move in opposite directions. However, atomic layers exhibit contour-phase motion for the modes 17.5 and $35.9 \mathrm{~cm}^{-1}$. The mode with the highest frequency of $178.3 \mathrm{~cm}^{-1}$ has mixed in-plane and out-of-plane character $\left(\mathrm{A}_{g}\right.$ like) with $\mathrm{Cd}$ and $\mathrm{Te}$ atoms having counter-phase motion.

Due to the heavier atomic masses and more ionic electronic character, phonon modes of single-layer CdTe lie at much lower energies than phonon modes of other 2D materials such as graphene, hBN and TMDs. Moreover, it was reported that phonon modes of structurally similar material of single-layer $\mathrm{ZnSe}$ lie at more higher energies than that of single-layer CdTe [24]. Thus, it is clear that single-layer CdTe is a quite soft material.

\section{Strain response of single-layer CdTe}

The built-in strain is inevitable as single-layer materials are usually grown on a substrate. It was shown that strain can significantly alter mechanical, electronic and magnetic properties of ultra-thin materials [51-53]. Thus, in this section the effects of out-of-plane compressive, in-plane compressive and tensile strains on the direct-gap semiconducting behavior of singlelayer CdTe are examined. The lattice constants of the unit cell 

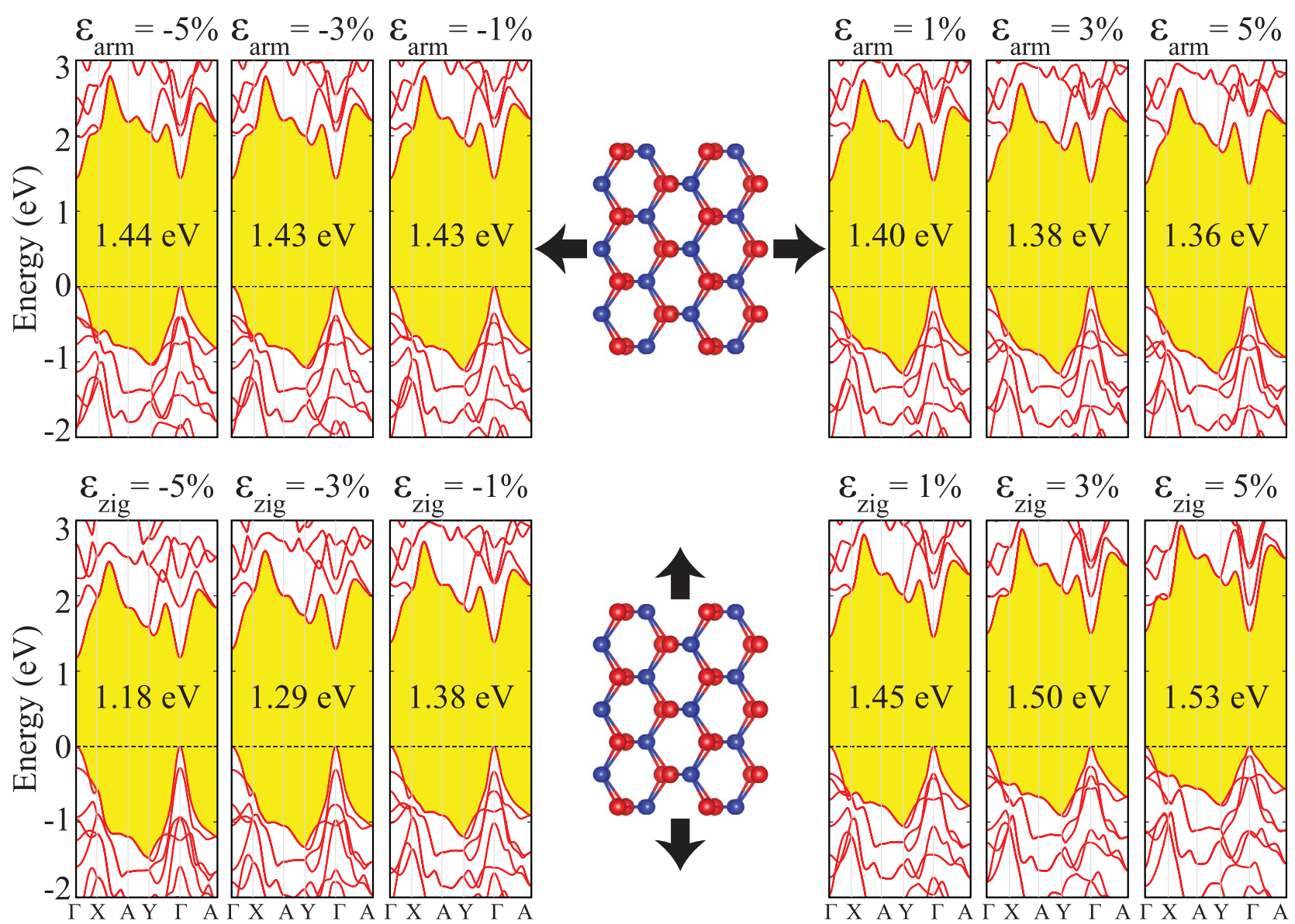

Figure 4. Evolution of the band dispersion of single-layer CdTe as a function of in-plane strain along armchair $\left(\varepsilon_{\text {arm }}\right)$ and zigzag $\left(\varepsilon_{\text {zig }}\right)$ directions. Fermi level is set to zero.

for in-plane compressive and tensile strains are changed up to $5 \%$ along zigzag (along $\vec{b}$ ) and armchair (along $\vec{a}$ ) directions. The thickness of the layer is compressed up to $5 \%$ for out-of-plane compressive strain calculations.

Figure 4 illustrates the evolution of electronic band structures for strained CdTe single-layer. It is clearly seen that electronic characteristics of single-layer CdTe do not change significantly with applied in-plane strain. It exhibits robust direct-gap at the $\Gamma$ point under considered strain values. It is found that the band gap of single-layer CdTe is more sensitive to the in-plane strain applied along zigzag direction than armchair direction. With the increase of tensile strain along the armchair direction, the band gap of CdTe decreases, whereas the band gap increases when compressive strain along the armchair direction is increased. However, the increase of tensile strain along zigzag direction results in an increase in the band gap of CdTe, the increase of compressive strain leads to decrease in the band gap. Figure 5 shows electronic band structures for single-layer of CdTe under compression along out-of-plane direction. It is found that CdTe does not show significant structural distortion under considered out-of-plane compression values. Direct gap character of $\mathrm{CdTe}$ at the $\Gamma$ point does not change, but the electronic band gap increases as applied compressive strain increases.

Variation of the band gap of single-layer CdTe crystal under out-of-plane and in-plane strains are shown in figure 6. It was already calculated that VBM of the CdTe is mainly composed of $p_{y}$ orbitals of Te atoms. Since the Te- $p_{y}$ orbitals are aligned in the zigzag direction, modification of band edges via applied strain occurs much faster than those in armchair direction. As shown in figure 6, while the band gap slowly decreases with increasing strain in armchair direction, it rapidly increases with increasing strain in zigzag direction.

Therefore, the variation of band gap of CdTe for applied tensile strain (in $\mp 5 \%$ ) along armchair direction is fitted to an expression as

$$
E_{\text {gap }}\left(\varepsilon_{\text {arm }}\right)=1.42-\alpha \varepsilon_{\text {arm }}-\beta \varepsilon_{\text {arm }}^{2}
$$

$\alpha$ and $\beta$ are fitting parameters and their values are $\sim 0.008$ and $0.001 \mathrm{eV}$. Compressive strain along the zigzag direction decreases the hybridization of $p_{y}$ orbitals of Te atom and $d$ orbitals of $\mathrm{Cd}$ atom at the VBM, whereas it increases the hybridization of Te and Cd orbitals at the CBM. Therefore, the VBM and CBM energies vary in opposite directions, thereby decreasing the band gap. The variation of band gap of singlelayer CdTe for applied tensile strain along zigzag direction is fitted to an expression as

$$
E_{\text {gap }}\left(\varepsilon_{\text {zig }}\right)=1.42+\gamma \varepsilon_{\text {zig }}-\delta \varepsilon_{\text {zig }}^{2}
$$

where $\gamma$ and $\delta$ are fitting parameters and their values are $\sim 0.035$ and $0.003 \mathrm{eV}$, respectively. As a result, strain-dependence of the band gap of single-layer CdTe exhibits nonlinear variations behavior when an in-plane strain is applied. 


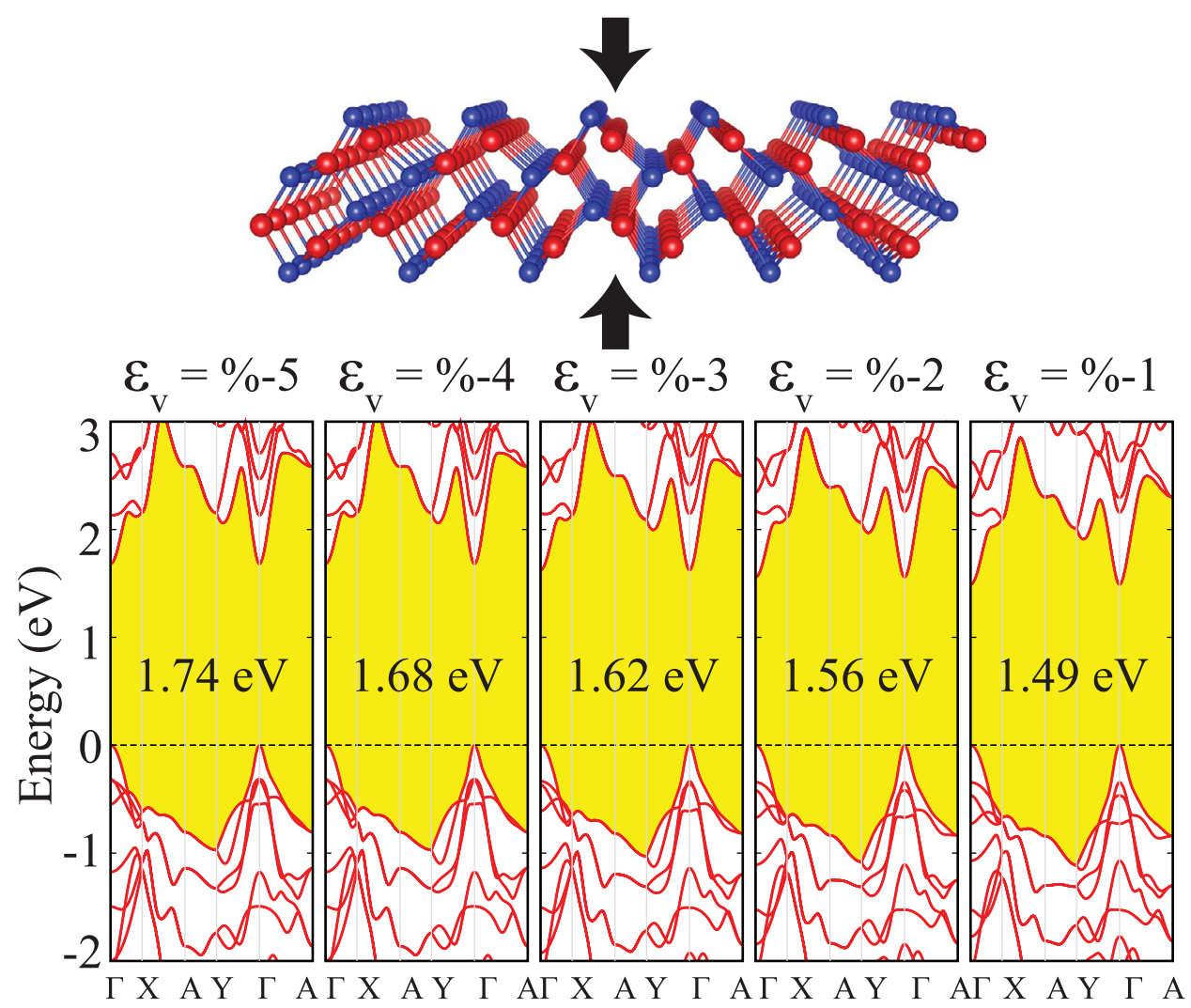

Figure 5. Evolution of the band dispersion of single-layer CdTe as a function of compressive strain along out-of-plane $\left(\varepsilon_{v}\right)$ direction. Fermi level is set to zero.

The out-of-plane strain application can easily alter the interlayer spacing of layered materials and therefore it provides an efficient way of tuning the electronic properties. In the section 3 we found that the CBM of CdTe is dominated by $p_{z}$ orbitals of Te atom. Therefore, application of compressive out-of-plane strain significantly affects the hybridization between orbitals of $\mathrm{Cd}$ and Te. Consequently, the band gap of CdTe increases monotonically with increasing compressive strain along out-of-plane direction and the rate of change for band gaps is faster for the out-of-plane strain than that of inplane strains. Increasing behavior of band gap of CdTe for applied compressive strain along out-of-plane direction is fitted to an expression as

$$
E_{\text {gap }}\left(\varepsilon_{v}\right)=1.42-\zeta \varepsilon_{v}
$$

$\zeta$ is a fitting parameter and it has a value of $\sim 0.065 \mathrm{eV}$.

It appears that while the direct band gap feature is maintained, controllable modification of the band gap values of monolayer CdTe is feasible by the application of uniaxial strain along different crystallographic orientations. Mostly, electronic properties of ultra-thin materials are highly sensitive to the applied strain. It was shown that strain changes the energy dispersion, band gap, and the band edges of graphene [54]. In another study, the optical band gap of $\mathrm{MoS}_{2}$ experiences a direct-to-indirect transition with applied strain, which decreases the measured photoluminescence intensity [55]. Previously we showed that electronic band structure of single-layer $\mathrm{MoSe}_{2}$ undergoes a direct to indirect band gap crossover under tensile strain [56]. Moreover, strain induced phase transition (from semiconducting $2 \mathrm{H}$ phase to metallic

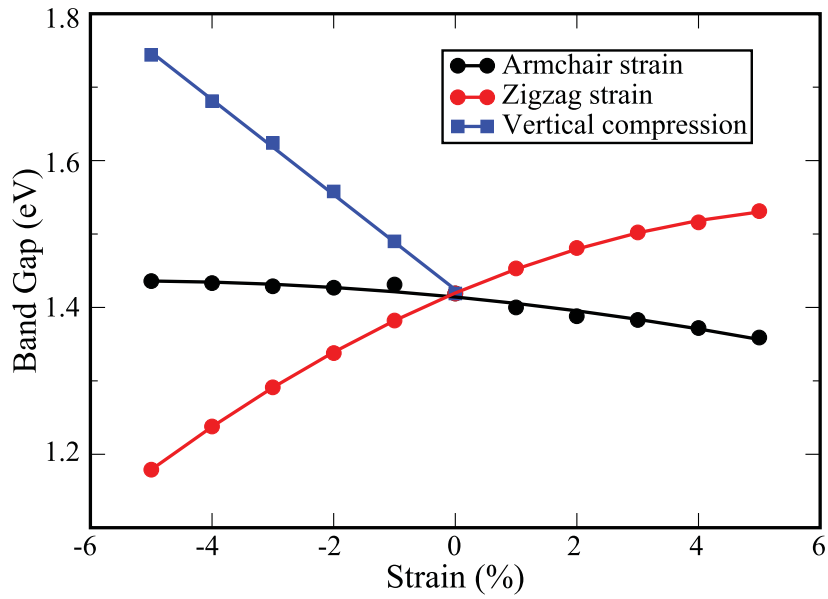

Figure 6. Evolution of band gaps of single-layer CdTe under inplane and out-of-plane strain. Dots are calculated values and lines are fitted values.

1T' phase) is observed in $\mathrm{MoTe}_{2}$ [57]. Therefore, in contrast to typical ultra-thin materials, monolayer CdTe exhibits robust and moderate band gap that covers the broad range of the solar spectrum, which are essential for its utilization in future electronics.

\section{Conclusions}

In this study, we investigated structural, phonon and electronic characteristics of single-layer CdTe by performing state-ofthe-art first principle calculations. Structural analysis revealed 
that ultra-thin CdTe has a crystal structure made of reconstructed 8-atomic primitive unit cell. Electronic band dispersion calculations showed that single-layer CdTe has a direct band gap of 1.42 (GGA) eV at the $\Gamma$ point. Direction dependent energy band dispersions at the vicinity of VBM and CBM indicate that single-layer CdTe has anisotropic electronic and optical properties.

Moreover, it is seen that electronic characteristics of singlelayer CdTe are more sensitive to in-plane strain applied along zigzag direction than armchair direction. Along the armchair direction, the higher the tensile strain, the smaller the bandgap. However, increasing the tensile strain along zigzag direction increases the band gap. In addition, when a compressive strain applied in out-of-plane direction, the rate of increase of the electronic bandgap is much faster. It is also found that the direct bandgap semiconducting behavior of the ultrathin CdTe is not affected by compressive and tensile strain applied in in-plane or out-of-plane directions. Ultra-thin CdTe crystal with its strain-independent and robust direct bandgap is quite suitable material for nanoscale optoelectronic device applications.

\section{Acknowledgments}

Computational resources were provided by TUBITAK ULAKBIM, High Performance and Grid Computing Center (TR-Grid e-Infrastructure). HS acknowledges financial support from the TUBITAK under the project number 116C073. HS acknowledges support from Bilim Akademisi-The Science Academy, Turkey under the BAGEP program.

\section{ORCID iDs}

F Iyikanat (1) https://orcid.org/0000-0003-1786-3235

J Kang (1) https://orcid.org/0000-0003-4788-0028

R T Senger (1) https://orcid.org/0000-0003-0800-1924

H Sahin (10 https://orcid.org/0000-0002-6189-6707

\section{References}

[1] Novoselov K S, Geim A K, Morosov S V, Jiang D, Katsnelson M I, Grigorieva I V, Dubonos S V and Firsov A A 2004 Science 306666

[2] Cahangirov S, Topsakal M, Akturk E, Sahin H and Ciraci S 2009 Phys. Rev. Lett. 102236804

[3] Bianco E, Butler S, Jiang S, Restrepo O D, Windl W and Goldberger J E 2013 ACS Nano 74414

[4] Vogt P, Padova P D, Quaresima C, Avila J, Frantzeskakis E, Asensio M C, Resta A, Ealet B and Lay G L 2012 Phys. Rev. Lett. 108155501

[5] Sahin H, Cahangirov S, Topsakal M, Bekaroglu E, Akturk E, Senger R T and Ciraci S 2009 Phys. Rev. B 80155453

[6] Zhu F-F, Chen W-J, Xu Y, Gao C-L, Guan D-D, Liu C-H, Qian D, Zhang S-C and Jia J-F 2015 Nat. Mater. 141020

[7] Rachel S and Ezawa M 2014 Phys. Rev. B 89195303

[8] Wang Q H, Zadeh K K, Kis A, Coleman J N and Strano M S 2012 Nat. Nanotechnol. 7699
[9] Esfahani D N, Leenaerts O, Sahin H, Partoens B and Peeters F M 2015 J. Phys. Chem. C 11910602

[10] Island J O, Kuc A, Diependaal E H, Bratschitsch R, van der Zant H S J, Heine T and Gomez A C 2016 Nanoscale 82589

[11] Chuang H-J, Chamlagain B, Koehler M, Perera M M, Yan J, Mandrus D, Tomanek D and Zhou Z 2016 Nano Lett. 161896

[12] Kang J, Sahin H and Peeters F M 2015 Phys. Chem. Chem. Phys. 1727742

[13] Late D J, Liu B, Luo J, Yan A, Matte H S S R, Grayson M, Rao C N R and Dravid V P 2012 Adv. Mater. 243549

[14] Cai H, Kang J, Sahin H, Chen B, Suslu A, Wu K, Peeters F M, Meng X and Tongay S 2016 Nanotechnolgy 27065203

[15] Yagmurcukardes M, Senger R T, Peeters F M and Sahin H 2016 Phys. Rev. B 94245407

[16] Mak K F, Lee C, Hone J, Shan J and Heinz T F 2010 Phys. Rev. Lett. 105136805

[17] Splendiani A, Sun L, Zhang Y, Li T, Kim J, Chim C-Y, Galli G and Wang F 2010 Nano Lett. 101271

[18] Radisavljevic B and Kis A 2013 Nat. Mater. 12815

[19] Radisavljevic B, Radenovic A, Brivio J, Giacometti V and Kis A 2011 Nature Nanotechnol. 6147

[20] Pu J, Yomogida Y, Liu K-K, Li L-J, Iwasa Y and Takenobu T 2012 Nano Lett. 124013

[21] Lee G-H et al 2013 ACS Nano 77931

[22] Bertolazzi S, Brivio J and Kis A 2011 ACS Nano 59703

[23] Tan C and Zhang H 2015 Nat. Commun. 67873

[24] Bacaksiz C, Senger R T and Sahin H 2017 Appl. Surf. Sci. 409426

[25] Ithurria S, Tessier M D, Mahler B, Lobo R P S M, Dubertret B and Efros A L 2011 Nat. Mater. 10936

[26] Park H, Chung H and Kim W 2013 Mater. Lett. 99172

[27] Son J S et al 2009 Angew. Chem. Int. Ed. 486861

[28] Sun Y, Sun Z, Gao S, Cheng H, Liu Q, Piao J, Yao T, Wu C, Hu S, Wei S and Xie Y 2012 Nat. Commun. 31057

[29] Rogalski A 2005 Rep. Prog. Phys. 682267

[30] Gupta A, Parikh V and Compaan A D 2006 Sol. Energy Mater. Sol. Cell 902263

[31] Tu C-C and Lin L Y 2008 Appl. Phys. Lett. 93163107

[32] Szeles C 2004 Phys. Status Solidi B 241783

[33] Ferekides C S, Balasubramanian U, Mamazza R, Viswanathan V, Zhao H and Morel D L 2004 Sol. Energy 77823

[34] Mahabaduge H P et al 2015 Appl. Phys. Lett. 106133501

[35] Green M A, Emery K, Hishikawa Y, Warta W and Dunlop E D 2016 Prog. Photovolt., Res. Appl. 24905

[36] Kim K-C, Kim H J, Suh S-H, Carmody M, Sivananthan S and Kim J-S 2010 J. Electron. Mater. 39863

[37] Diamant R, Ponce L, Fernandez M and Jimenez E 1998 Appl. Phys. B 66639

[38] Bonilla S and Dalchiele E A 1991 Thin Solid Films 204397

[39] Ison V V, Rao A R and Dutta V 2009 Solid State Sci. 112003

[40] Wang J, Meng J, Li Q and Yang J 2016 Phys. Chem. Chem. Phys. 1817029

[41] Zheng H, Li X-B, Chen N-K, Xie S-Y, Tian W Q, Chen Y, Xia H, Zhang S B and Sun H-B 2015 Phys. Rev. B 92115307

[42] Kresse G and Joubert D 1999 Phys. Rev. B 591758

[43] Kresse G and Furthmuller J 1996 Phys. Rev. B 5411169

[44] Perdew J P, Burke K and Ernzerhof M 1996 Phys. Rev. Lett. 773865

[45] Henkelman G, Arnaldsson A and Jonsson H 2006 Comput. Mater. Sci. 36354

[46] Alfè D 2009 Comput. Phys. Commun. 1802622

[47] Groiss H, Hesser G, Heiss W, Schäffler F, Leitsmann R, Bechstedt F, Koike K and Yano M 2009 Phys. Rev. B 79235331 
[48] Guo L, Zhang S, Feng W, Hu G and Li W 2013 J. Alloys Compd. 579583

[49] Amirtharaj A and Pollak F H 1984 Appl. Phys. Lett. 45789

[50] Zitter R N 1971 Surf. Sci. 28335

[51] Rodin A S, Carvalho A and Neto A H C 2014 Phys. Rev. Lett. 112176801

[52] Jiang J-W and Park H-S 2014 Nat. Comm. 54727

[53] Tao P, Guo H, Yang T and Zhang Z 2014 J. Appl. Phys. 115054305
[54] Wong J-H, Wu B-R and Lin M-F 2012 J. Phys. Chem. C 1168271

[55] Conley H J, Wang B, Ziegler J I, Haglund R F J, Pantelides S T and Bolotin K I 2013 Nano Lett. 133626

[56] Horzum S, Sahin H, Cahangirov S, Cudazzo P, Rubio A, Serin T and Peeters F M 2013 Phys. Rev. B 87125415

[57] Song S, Keum D H, Cho S, Perello D, Kim Y and Lee Y H 2016 Nano Lett. 16188 